\title{
The effect of the number of anchors used in the medial row on clinical outcomes in arthroscopic double row repair of rotator cuff tears: retrospective evaluation of patients with 3 to 7 years of follow-up
}

\author{
๑Bertan Cengiz \\ Acıbadem Kayseri Hospital, Department of Orthopaedics and Traumatology, Kayseri, Turkey
}

Cite this article as: Cengiz B. The effect of the number of anchors used in the medial row on clinical outcomes in arthroscopic double row repair of rotator cuff tears: retrospective evaluation of patients with 3 to 7 years of follow-up. J Health Sci Med 2022; 5(1): 331-335.

\begin{abstract}
Aim: Arthroscopic double row (DR) suture anchor repair is one of the most frequently applied methods in the surgical treatment of rotator cuff tear (RCT). Various modifications have been tried to eliminate some of the disadvantages of this technique such as operation time, high cost, and the high risk of retearing. In this study, we aimed to investigate whether placing a single or double suture anchor in the medial row affects clinical and functional outcomes and retear rates in patients with RCT who were operated with the DR suture anchor technique.

Material and Method: A retrospective study including 58 patients aged 18-65 years who underwent DR suture anchor repair due to medium-sized RCT and had a minimum follow-up period of 3 years was conducted. One knotless anchor was placed in the lateral row in all patients. In the medial row, we placed 1 all suture anchor (ASA) in group 1 and 2 ASAs in group 2. Visual pain scale (VAS), University of California Los Angeles Score (UCLA), American shoulder and elbow score (ASES) scales were used for preoperative and postoperative clinical and functional evaluation, and complications and retears were recorded.

Results: When the preoperative and postoperative VAS, UCLA and ASES scores were compared within groups, there were significant difference from pre- to post-operative findings in both groups $(\mathrm{p}<0.001)$. However, there was no statistically significant difference between the groups in terms of preoperative and postoperative VAS, UCLA and ASES score ( $p>0.05$ ). Operation time in Group 1 was significantly shorter than in Group 2 ( $\mathrm{p}<0.001$ ).

Conclusion: In DR suture anchor repair, there is no difference in clinical outcomes and retear rates in surgeries utilizing single or double suture anchor placement in the medial row. Increasing the number of anchors in the medial row does not contribute to clinical and functional results, and has disadvantages such as increasing operation time and cost.
\end{abstract}

Keywords: Rotator cuff tear, double row repair, retear, UCLA, ASES

\section{INTRODUCTION}

Rotator cuff tear (RCT) is the most common cause of shoulder pain in adults (1). Surgical treatment of RCTs, which can cause severe pain and weakness that limit daily activities and significantly hinder quality of life, has been applied for years, but procedural improvements are being made continuously $(2,3)$.

Arthroscopic surgery has various advantages including reduced postoperative morbidity, pain and deltoid dysfunction, and therefore, is currently accepted as the gold standard treatment in rotator cuff repair (3). Among the various arthroscopic repair models, the most commonly used are single row (SR) and double row (DR) suture anchor techniques. The main difference of $\mathrm{DR}$ repair from SR repair is a second anchor series placed on the medial aspect of the tendon -in addition to the fact that anchors are placed on the lateral side of the tendon footprint (4). It was thought that with this technique, a wider restoration of the anatomical footprint could be achieved, the repair strength would be higher, less stress would occur on the anchors and knots, and better healing rates could be achieved (3). However, there is still no consensus on which technique achieves the best clinical and functional results (5-8). On the other hand, despite many advantages of arthroscopic repair, higher retear rates have been reported with these techniques $(9,10)$. For this reason, studies are continuing on different modifications to increase clinical 
efficiency, reduce complication rates, shorten operation time and increase cost effectiveness.

In this study, we aimed to investigate whether the number of sutures applied in the medial row had an effect on clinical outcomes and retearing rates in DR suture repair, which is one of the most commonly used techniques for RCT repair.

\section{MATERIAL AND METHOD}

In our retrospective study, the patient records of 329 patients who underwent arthroscopic RCT surgery in our hospital between 2014-2018 were evaluated. The study was carried out with the permission of the Acibadem University Ethics Committee (Date: 14.10.2021, Decision No: 2021-20/44). All procedures were carried out in accordance with the ethical rules and the principles of the Declaration of Helsinki.

\section{Patients}

The inclusion criteria were as follows: being aged 18-65 years, having medium sized RCT $(1-3 \mathrm{~cm})$ diagnosed by magnetic resonance imaging (MRI) according to the Deorio and Cofield classification, having undergone treatment with DR suture anchor repair, and attending follow-up studies for at least three years (11). Patients younger than 18 and those older than 65 years, those with a tear greater than $3 \mathrm{~cm}$ or less than $1 \mathrm{~cm}$, those with a history of previous shoulder surgery, those who received steroid injections within the last 3 months, subjects who underwent SR cuff repair, patients who had DR cuff repair but had titanium suture anchor for the medial row or knotted suture anchor for the lateral row, those who had insufficient follow-up time, and individuals who had undergone revision surgery were excluded from the study.

Of the 329 patients examined, 58 patients who met these criteria were divided into 2 groups: Group $1(\mathrm{n}=31)$ : 1 double-loaded four-strand all-suture anchor (ASA) for the medial row and 1 Peek anchor for the lateral row, and Group $2(\mathrm{n}=27)$ : patients with 2 double-loaded four-strand all-suture anchors for the medial row and 1 Peek anchor for the lateral row.

\section{Procedure}

If the tear involved only the central supraspinatus region (anterior or posterior portions intact), or if it was $\mathrm{U}$ or $\mathrm{V}$ shaped, or if the retraction was less than $1 \mathrm{~cm}$, we used only 1 medial anchor. If the tear involved the anterior or posterior of the supraspinatus, and therefore, the central tendon was displaced anteriorly or posteriorly by medial retraction (L-shape, reverse L-shape or Trapezoidal shape), or if the tear included the infraspinatus, or if there was a retraction of $\geq 1 \mathrm{~cm}$ (in short, if the patient had a more complicated and larger tear), 2 medial anchors were used.
In patients, we used double-loaded four-strand ASA (2.9 $\mathrm{mm}$; JuggerKnot ${ }^{\oplus}$, Biomet Inc., Warsaw, IN, USA) for the medial row, while the knotless anchor was used for the lateral row (3.5 or 4.5 mm PushLock ${ }^{\oplus}$ anchor, Arthrex ${ }^{\oplus}$, Naples, FL, USA).

In the clinical and functional evaluation of the patients, preoperative and postoperative (mean $56.9 \pm 12.9$ months) visual analog scale (VAS), University of California at Los Angeles Score (UCLA), American Shoulder and Elbow Score (ASES) scales were used. In addition, complications and retears were recorded.

The VAS is the most widely utilized and easy to use pain rating scale (12). Pain is scored subjectively between a minimum of 0 (none) and a maximum of 10 (worst imaginable pain). The UCLA is a 35 -point self-report scale assessing patients based on the pain, function, strength, movement and patient satisfaction subdimensions (13). The ASES is a 100-point self-report scale consisting of two sub-headings that evaluate pain and 10 different activities related to daily life (14).

\section{Surgical Technique}

The surgical procedure was performed under general anesthesia in the lateral decubitus position. All patients were operated by a single surgeon. Standard arthroscopic portals were opened; the posterolateral portal was used as the imaging portal, and the anterolateral and lateral portals were used as the working portal. First of all, other pathologies of the joint were detected by diagnostic arthroscopy. The biceps tendon was evaluated, and if there was a pathology of the tendon, necessary intervention was performed (tenodesis or tenotomy) according to the patient's age/size of the lesion/condition of the tendon. Acromioplasty was performed on each patient as a standard approach. After the tear was detected, the size of the tear was determined using a probe. The degenerate and fringing parts were debrided, followed by preparation of the footprint. During surgery, the medial row anchors were placed medially, closest to the chondral junction. A double-loaded four-strand ASA was used as the medial row anchor. According to the shape and size of the tear, the quality of the tendon and how much it could be mobilized, 1 or 2 ASAs were used. All threads of the anchor were threaded with the help of a suture threader, approximately $10 \mathrm{~mm}$ medial to the tear, leaving the appropriate distance between each suture. Then the medial row threads were knotted. If 1 ASA was used for the medial row, both knotted threads were loaded on the knotless anchor (PushLock) and placed $1-1.5 \mathrm{~cm}$ lateral to the greater tuberculum as lateral row anchors. If 2 ASAs were used, all threads were knotted in the medial row by passing through the rotator cuff, then all sutures were loaded onto the PushLock and placed as lateral row anchors. 


\section{Postoperative Rehabilitation}

A shoulder abduction splint was used for 6 weeks in the postoperative period. Mild passive forward flexion exercises were started on the 2 nd postoperative day. After 6-8 weeks, active assisted range of motion (ROM), active ROM and strengthening exercises were started gradually. Light activities were allowed after 3 months, and heavy activities and sports were allowed after 9 months.

\section{Statistical Analysis}

The SPSS version 18.0 Windows software (SPSS Inc., Chicago, IL, USA) was used for the statistical analysis of obtained data. Categorical variables were compared using chi-squared tests. The Shapiro-Wilk test was used to determine normality of distribution. The comparison of quantitative differences between the groups was assessed with the Student's t-test or the Mann-Whitney test, depending on normality of distribution. The nonparametric Wilcoxon Signed Ranks test was used to test for differences between related (paired) samples. Differences with a $\mathrm{p}$ value of $<0.05$ were considered to be statistically significant.

\section{RESULTS}

Of the 329 examined patients, 58 were eligible for the study according to aforementioned criteria (Figure). The median (IQR) age of the patients was 58 (51-64) years; 26 were female and 32 were male. There was no significant difference between the two groups in terms of demographic characteristics, operated side and retear rates $(\mathrm{p}>0.05)$. While the operation time was $62.19 \pm 6.25$ minutes in Group 1, it was $72.22 \pm 4.98$ minutes in Group 2. There was a significant difference between the two groups in terms of operation time $(\mathrm{p}<$ 0.001) (Table 1).

\begin{tabular}{|c|c|c|c|c|}
\hline & \multicolumn{2}{|c|}{$\begin{array}{l}\text { Number of all-suture } \\
\text { anchors }\end{array}$} & \multirow[b]{2}{*}{ All } & \\
\hline & One $(n=31)$ & Two $(n=27)$ & & \\
\hline \multicolumn{5}{|l|}{ Sex } \\
\hline Female & $14(45.2 \%)$ & $12(44.4 \%)$ & $26(44.8 \%)$ & 0.956 \\
\hline Male & $17(54.8 \%)$ & $15(55.6 \%)$ & $32(55.2 \%)$ & \\
\hline Age (year) & $57(50-62)$ & $59(53-64)$ & $58(51-64)$ & 0.425 \\
\hline \multicolumn{5}{|l|}{ Side } \\
\hline Left & $15(48.4 \%)$ & $13(48.1 \%)$ & $28(48.3 \%)$ & 0.986 \\
\hline Right & $16(51.6 \%)$ & $14(51.9 \%)$ & $30(51.7 \%)$ & \\
\hline $\begin{array}{l}\text { Operation time } \\
\text { (mins) }\end{array}$ & $62.19 \pm 6.25$ & $72.22 \pm 4.98$ & $66.86 \pm 7.57$ & $<0.001$ \\
\hline $\begin{array}{l}\text { Follow-up time } \\
\text { (months) }\end{array}$ & $55.68 \pm 13.3$ & $58.22 \pm 12.56$ & $56.86 \pm 12.91$ & 0.459 \\
\hline Retear & $3(9.7 \%)$ & $3(11.1 \%)$ & $6(10.3 \%)$ & 0.858 \\
\hline \multicolumn{5}{|c|}{$\begin{array}{l}\text { Data are given as mean } \pm \text { standard deviation or median (1st quartile-3rd quartile) } \\
\text { for continuous variables according to normality of distribution, and frequency } \\
\text { (percentage) for categorical variables }\end{array}$} \\
\hline
\end{tabular}

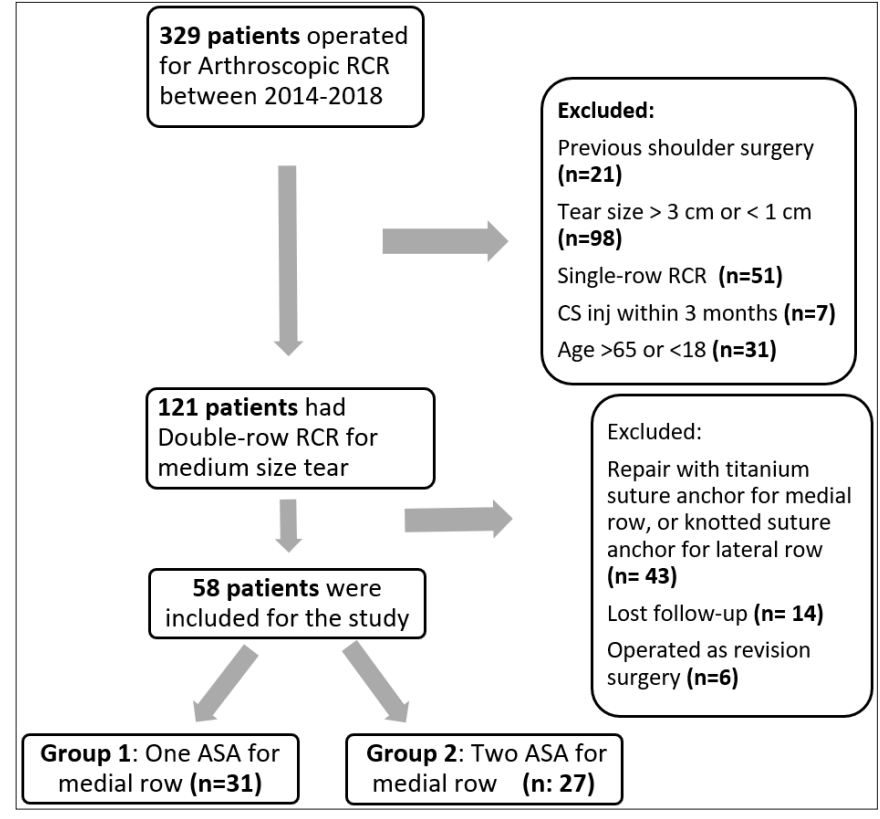

Figure. Flowchart of the study

RCR, Rotator cuff repair; CS, Corticosteroid; ASA, All-suture anchor.

A significant difference was found between the preoperative and postoperative ASES, UCLA and VAS scores of patients in both groups $(\mathrm{p}<0.001)$. When group 1 and group 2 were compared in terms of preoperative and postoperative VAS, UCLA and ASES scores, no significant difference was found in any parameter $(\mathrm{p}>$ 0.05) (Table 2).

\begin{tabular}{|c|c|c|c|}
\hline & \multicolumn{2}{|c|}{ Number of all-suture anchors } & \multirow{2}{*}{$\mathbf{p}^{*}$} \\
\hline & One $(n=31)$ & Two $(n=27)$ & \\
\hline \multicolumn{4}{|l|}{ ASES score } \\
\hline Preoperative & $40(36-42)$ & $42(38-46)$ & 0.280 \\
\hline Last follow-up & $88(86-92)$ & $90(88-92)$ & $0.64 \mathrm{c}$ \\
\hline $\mathrm{p}^{* *}$ & $<0.001$ & $<0.001$ & \\
\hline \multicolumn{4}{|l|}{ UCLA score } \\
\hline Preoperative & $14(12-16)$ & $14(12-16)$ & $0.34 \mathrm{c}$ \\
\hline Last follow-up & $32(28-32)$ & $32(30-34)$ & 0.107 \\
\hline $\mathrm{p}^{* *}$ & $<0.001$ & $<0.001$ & \\
\hline \multicolumn{4}{|l|}{ VAS score } \\
\hline Preoperative & $8(7-8)$ & $8(7-8)$ & $0.37 \mathrm{c}$ \\
\hline Last follow-up & $3(2-3)$ & $2(2-4)$ & $0.57 \mathrm{c}$ \\
\hline $\mathrm{p}^{\star *}$ & $<0.001$ & $<0.001$ & \\
\hline
\end{tabular}

\section{DISCUSSION}

In our retrospective study, in which we compared two different modifications of the arthroscopic DR suture anchor rotator cuff repair technique, we did not find significant differences in clinical outcomes and complication rates in the two groups. However, the operation time was significantly shorter in recipients of a single suture anchor to the medial row. 
Although there are conflicting results in studies comparing DR and SR repair methods, it is generally accepted that DR repair is more effective in large and massive tears $(8,15-21)$. In their study comparing DR and SR repair in medium and large-sized tears, Koh et al. found no significant difference in clinical outcomes and complication rates between the two groups. Of note, one suture anchor was applied to the medial row in the patient group who underwent DR repair (17). Similarly, in our study, 1 suture anchor was applied to the medial row in Group 1, but unlike the mentioned study, the other group underwent repair via DR with 2 suture anchors, rather than SR. Nonetheless, our results also showed similar outcomes, but it must be noted that all patients in our study had a medium-sized tear according to the Deorio and Cofield classification.

Despite the successful results of suture anchor repair, there is still a risk of retear reported at varying rates (20$90 \%)$ in the literature (23). Although the risk of retear appears to be greater in SR suture anchor repair, retear risk is a general problem in arthroscopic rotator cuff repairs (24). Methods that can increase repair strength are necessary to reduce the risk of retearing (25). In some studies, the musculotendinous junction has been shown as the primary point of failure in patients who underwent DR repair (26). Revision of this region is difficult and the medial row should not be overstretched to avoid it (3). Medial insufficiency due to excessive load and tendon strangulation in the medial knots necessitates preventive measures to improve medial row integrity (3). In this study, we hypothesized that using changing the number of medial row sutures could be associated with medial row overstretching and medial insufficiency development. However, we did not find a significant difference between the two groups in terms of retear rates. The fact that our retear rates were low overall $(10.3 \%)$ compared to the literature may be due to the small number of patients in the study and the inclusion of only medium-sized RCT cases.

Depending on the suture anchor repair, there are other problems such as dislodgement of the anchor, impingement of the knot, difficulty in revision and high costs (27). In order to overcome these problems, studies are carried out on different techniques which employ various approaches, including reducing the number of anchors, repairing without anchors, reducing the number of sutures, or changing the configuration (2730). The DR technique also has other disadvantages, such as prolonged operation time, steep learning curve, and the fact that it is a relatively complicated and expensive procedure $(8,15-18)$. Various studies have been carried out on different modifications of the DR technique in order to shorten operation time, simplify the procedure (albeit partially), and to reduce complication rates. In a recent review, out of 9 studies comparing different DR configurations, none showed a significant superiority over the others in terms of clinical outcomes (22), indicating that these modifications have had little effect on outcomes. It is known that increased duration of surgical operations is associated with increased risk of infection, transfusion likelihood and thrombosis development (31). As mentioned above, since there no significant differences have been reported between clinical and functional results in different arthroscopic rotator cuff surgery models, complications and costs gain importance in addition to various factors determining the choice of surgical approach. In our study, operation times were significantly lower in single anchor recipients. In this respect, our study results can be seen as preliminary data for more detailed studies aimed at reducing the costs and possible complications related to prolonged operation times.

It has been suggested that increased operative time may also have an effect on the retear rates, and various studies have been conducted to seek possible relationships. Le et al. found that longer operative time was correlated with increased retear rate (32). In another study, authors found that the duration of surgery did not have a significant independent effect on rotator cuff retear rates at the postoperative 6th month (31). In our study, while there was a significant difference between the two groups in terms of operation time, there was no significant difference between the retear rates.

Our study has some limitations such as its retrospective design and the relatively low number of patients. The fact that the study was single-centered and only included non-severe tears also limit generalizability. In addition, since the decision for surgical approach (exposure) was based on a pre-defined protocol according to patientand tear-based characteristics, outcomes could have been influenced by differences in management approaches and post-operative care throughout the study period. Therefore, larger-scale multicenter prospective studies would be more beneficial to investigate the advantages and disadvantages of these different modifications.

\section{CONCLUSION}

In DR suture anchor repair of rotator cuff tears, increasing the number of anchors in the medial row does not contribute to clinical and functional results; in fact, this approach has disadvantages such as increasing operation time and costs. There is a need for prospective studies with a larger number of patients with better categorization. 


\section{ETHICAL DECLARATIONS}

Ethics Committee Approval: The study was carried out with the permission of the Acibadem University Ethics Committee (Date: 14.10.2021, Decision No: 2021-20/44).

Informed Consent: Because the study was designed retrospectively, no written informed consent form was obtained from patients

Referee Evaluation Process: Externally peer-reviewed.

Conflict of Interest Statement: The authors have no conflicts of interest to declare.

Financial Disclosure: The authors declared that this study has received no financial support.

Author Contributions: All of the authors declare that they have all participated in the design, execution, and analysis of the paper and that they have approved the final version.

\section{REFERENCES}

1. Kim KC, Shin HD, Lee WY, Yeon KW, Han SC. Clinical outcomes and repair integrity of arthroscopic rotator cuff repair using suture-bridge technique with or without medial tying: prospective comparative study. J Orthop Surg Res 2018; 13: 212.

2. Kitagaki JJ, Gomes G, Teruo F, Archetti N, Pozzetti J, Sugawara MJ. Functional outcomes of traumatic and non-traumatic rotator cuff tears after arthroscopic repair. World J Orthop 2017; 8: 631-7.

3. Aydin N, Karaismailoglu B, Gurcan M, Ozsahin MK. Arthroscopic double-row rotator cuff repair: a comprehensive review of the literature. SICOT J 2018; 4: 57.

4. Lo IK, Burkhart SS. Double-row arthroscopic rotator cuff repair: re-establishing the footprint of the rotator cuff. Arthroscopy 2003; 19: 1035-42.

5. Plachel F, Siegert P, Rüttershoff $\mathrm{K}$, et al. Long-term results of arthroscopic rotator cuff repair: a follow-up study comparing single-row versus double-row fixation techniques. Am J Sports Med 2020; 48: 1568-74.

6. Abrams JS. Editorial commentary: improving strength and clinical outcomes after rotator cuff repair-role of choosing singleversus double-row repair. Arthroscopy 2019; 35: 2756-8.

7. Yoon JS, Kim SJ, Choi YR, Kim SH, Chun YM. arthroscopic repair of the isolated subscapularis full-thickness tear: single- versus double-row suture-bridge technique. Am J Sports Med 2019; 47: 1427-33.

8. Franceschi F, Ruzzini L, Longo UG. Equivalent clinical results of arthroscopic single-row and double-row suture anchor repair for rotator cuff tears: a randomized controlled trial. Am J Sports Med. 2007; 35: 1254-60.

9. Bedeir YH, Schumaier AP, Abu-Sheasha G, Grawe BM. Type 2 retear after arthroscopic single-row, double-row and suture bridge rotator cuff repair: a systematic review. Eur J Orthop Surg Traumatol 2019 29: 373-82.

10. Rossi LA, Chahla J, Verma NN, Millett PJ, Ranalletta M. Rotator cuff retears. JBJS Rev 2020; 8: e0039.

11.De Orio JK, Cofield RH. Results of a second attempt at surgical repair of a failed initial rotator-cuff repair. J Bone Joint Surg Am 1984; 66: 563-7.

12. Jensen MP, Karoly P, Braver S. The measurement of clinical pain intensity: a comparison of six methods. Pain. 1986; 27: 117-26.

13. Ellman H, Hanker G, Bayer M. Repair of the rotator cuff. Endresult study of factors influencing reconstruction. J Bone Joint Surg Am 1986; 68: 1136-44.
14. Richards RR, An KN, Bigliani LU, et al. A standardized method for the assessment of shoulder function. J Shoulder Elbow Surg 1994; 3: 347-52.

15. Burks RT, Crim J, Brown N, et al. A prospective randomized clinical trial comparing arthroscopic single- and double-row rotator cuff repair: magnetic resonance imaging and early clinical evaluation. Am J Sports Med 2009; 37: 674-82.

16. Grasso A, Milano G, Salvatore M, et al. Single-row versus doublerow arthroscopic rotator cuff repair: a prospective randomized clinical study. Arthrosc J Arthrosc Relat Surg 2009; 25: 4-12.

17. Koh KH, Kang KC, Lim TK, et al. Prospective randomized clinical trial of single- versus double-row suture anchor repair in 2- to $4-\mathrm{cm}$ rotator cuff tears: clinical and magnetic resonance imaging results. Arthroscopy 2011; 27: 453-62.

18. Aydin N, Kocaoglu B, Guven O. Single-row versus double-row arthroscopic rotator cuff repair in small- to medium-sized tears. J Shoulder Elbow Surg 2010; 19: 722-5.

19.Lapner P, Li A, Pollock JW, et al. A Multicenter randomized controlled trial comparing single-row with double-row fixation in arthroscopic rotator cuff repair: long-term follow-up. Am J Sports Med 2021; 49: 3021-9.

20. Sobhy MH, Khater AH, Hassan MR, El Shazly O. Do functional outcomes and cuff integrity correlate after single- versus doublerow rotator cuff repair? a systematic review and meta-analysis study. Eur J Orthop Surg Traumatol 2018; 28: 593-605.

21. Khoriati AA, Antonios T, Gulihar A, Singh B. Single vs double row repair in rotator cuff tears-a review and analysis of current evidence. J Clin Orthop Trauma 2019; 10: 236-40.

22. Rossi LA, Rodeo SA, Chahla J, Ranalletta M. Current concepts in rotator cuff repair techniques: biomechanical, functional, and structural outcomes. Orthop J Sports Med 2019; 7: 2325967119868674

23.Wu X, Briggs L, Murrell GA. Intraoperative determinants of rotator cuff repair integrity an analysis of 500 consecutive repairs. Am J Sports Med 2012; 40 : 2771-6.

24. Millett PJ, Warth RJ, Dornan GJ, Lee JT, Spiegl UJ. Clinical and structural outcomes after arthroscopic single-row versus doublerow rotator cuff repair: a systematic review and meta-analysis of level I randomized clinical trials. J Shoulder Elbow Surg 2014; 23: 586-97.

25. Wang VM, Wang FC, McNickle AG, et al. Medial versus lateral supraspinatus tendon properties: implications for double-row rotator cuff repair. Am J Sports Med 2010; 38: 2456-63.

26. Kaplan K, Elattrache NS, Vazquez O, et al. Knotless rotator cuff repair in an external rotation model: the importance of medialrow horizontal mattress sutures. Arthroscopy 2011; 27: 471-8.

27. Kuroda S, Ishige N, Mikasa VM. Advantages of arthroscopic trans osseous suture repair of the rotator cuff without the use of anchors. Clin Orthop Relat Res. 2013; 471: 3514-22.

28. Lee KW, Seo DW, Bae KW, Choy WS. Clinical and radiological evaluation after arthroscopic rotator cuff repair using suture bridge technique. Clin Orthop Surg 2013; 5: 306-13.

29. Takeuchi Y, Sugaya H, Takahashi N, et al. Repair integrity and retear pattern after arthroscopic medial knot-tying after suturebridge lateral row rotator cuff repair. Am J Sports Med 2020; 48: 2510-7.

30. Randelli P, Stoppani CA, Zaolino C, Menon A, Randelli F, Cabitza P. Advantages of arthroscopic rotator cuff repair with a trans osseous suture technique: a prospective randomized controlled trial. Am J Sports Med 2017; 45: 2000-9.

31. Elkins AR, Lam PH, Murrell GAC. Duration of surgery and learning curve affect rotator cuff repair retear rates: a post hoc analysis of 1600 cases. Orthop J Sports Med 2020; 8: 2325967120954341.

32.Le BT, Wu XL, Lam PH, Murrell GA. Factors predicting rotator cuff retears: an analysis of 1000 consecutive rotator cuff repairs. Am J Sports Med 2014; 42: 1134-42. 